\title{
Isolation of Gemella morbillorum in Herniated Intervertebral Disc Tissue in a Lumbar Discectomy Patient: A Case Report
}

\author{
Niroshima Dedunu Withanage ${ }^{1}$, Lohini Vijayendran Athiththan ${ }^{2 *}$, Sunil Perera ${ }^{3}$, \\ Sujatha Pathirage ${ }^{4}$, Hemantha Peiris ${ }^{2}$
}

\author{
${ }^{1}$ Department of Allied Health Sciences, Faculty of Medical Sciences, University of Sri Jayewardenepura, Nugegoda, Sri Lanka \\ ${ }^{2}$ Department of Biochemistry, Faculty of Medical Sciences, University of Sri Jayewardenepura, Nugegoda, Sri Lanka \\ ${ }^{3}$ The Central Hospital, Colombo, Sri Lanka \\ ${ }^{4}$ Medical Research Institute, Colombo, Sri Lanka \\ Email: *lohinia@sjp.ac.lk
}

\begin{abstract}
How to cite this paper: Withanage, N.D., Athiththan, L.V., Perera, S., Pathirage, S. and Peiris, H. (2017) Isolation of Gemella morbillorum in Herniated Intervertebral Disc Tissue in a Lumbar Discectomy Patient: A Case Report. Journal of Biosciences and Medicines, 5, 99-104.

https://doi.org/10.4236/jbm.2017.53010
\end{abstract}

Received: February 16, 2017

Accepted: March 20, 2017

Published: March 23, 2017

Copyright $\odot 2017$ by authors and Scientific Research Publishing Inc. This work is licensed under the Creative Commons Attribution International License (CC BY 4.0).

http://creativecommons.org/licenses/by/4.0/

\begin{abstract}
Back pain associated with lumbar disc herniation is a common musculoskeletal disorder that leads to absence at work place worldwide. Studies have proven in addition to the traditional factors, microbes play a role in disc herniation causing chronic back pain. A 34-year-old male who has not involved in any traumatic work but has a family history of disc herniation presented with lower back pain and numbness in his right leg. He had previously undergone lumbar discectomy at L4/L5 ten years back. Magnetic Resonance Imaging (MRI) showed L5/S1 right para central disc herniation impinging on the right S1 and S2 nerve roots. Standard protocols for disinfection of instruments, external skin and all transport media were adhered. Skin scrapings, muscle biopsy and excised disc tissue were obtained for anaerobic and aerobic bacterial cultures. Anaerobic microbial cultures of excised disc tissue following lumbar discectomy showed Gram positive growth. Further anaerobic isolation carried out using RapID ANA ID kit confirmed the growth as Gemella morbillorum. In addition, neither of the control samples (muscle nor skin) had any anaerobic growth indicating the absence of contamination. Aerobic bacterial growth was not present in the skin, muscles and disc cultures. The study findings add to the available literature, on the role of microorganisms in lumbar disc herniation and future treatment regimens with antibiotics.
\end{abstract}

\section{Keywords}

Lumbar Disc Herniation, Intervertebral Disc, Anaerobic Bacteria, Gemella morbillorum 


\section{Introduction}

The main causative factor for lower back pain is lumbar disc herniation. Other disorders such as osteoarthritis, ankylosing spondylitis, tuberculosis and osteoporosis also contribute to lower back pain. Studies have stated that the prevalence of disc prolapse in European countries is about 1\% - 3\% [1]. Even though the etiology of lumbar disc disease is poorly understood, multifactor such as physical loading, driving heavy motor vehicles, vibrations, smoking and strenuous sporting activities have been attributed to lumbar disc disease. But recent studies have indicated the involvement of microorganism and genetic factors as additional factors [2] [3]. Many studies have stated the involvement of Propionibacterium acnes in lumbar disc herniated patients [2] [3]. Hence this study was conducted to identify the presence of microorganisms in patients undergoing lumbar discectomy.

\section{Case Report}

\subsection{Clinical Presentation and Neuroimaging}

A 34-year-old male, who had not engaged in traumatic work but had a family history of disc herniation, presented to the clinic with lower back pain and numbness in his right leg for 6 months. He had difficulty in walking with sensory impairment in lateral side of the right leg. L5/S1 right para central disc herniation impinging on the right S1 and S2 nerve roots was identified with the aid of Magnetic Resonance Imaging (MRI) as shown in Figure 1. He had a past surgical history of L4/L5 microdiscectomy in 2005.

Informed written consent was obtained from the patient for publication.

\subsection{Sample Collection}

Standard protocols for disinfection of instruments, external skin and all transport media were adhered. During L5/S1 lumbar discectomy, surgically excised intervertebral disc, skin scrapings and muscle biopsy were taken for anaerobic and aerobic bacterial cultures.

\subsection{Identification of the Microbial Species}

Excised disc material, skin and muscles were investigated for the presence of aerobic and anaerobic bacteria. Skin scrapings and muscle biopsy samples were used as controls, to rule out the possibility of contamination. Bacterial cultures were further subjected to Gram stain and RapID ANA II ID kit.

In aerobic isolation, no aerobes were identified in the disc, skin and muscles tissues. Anaerobic disc culture plates, when read at day 2 and 7, showed Gram positive growth of anaerobe. This was further identified using RapID ANA II ID kit (remel, USA) and was confirmed as Gemella morbillorum. Probability of isolation of Gemella morbillorum in the positive culture $>99.9 \%$ (according to the microcode 020751 of the kit analysis). However the control samples skin and muscles did not have any growth for anaerobic isolation. 


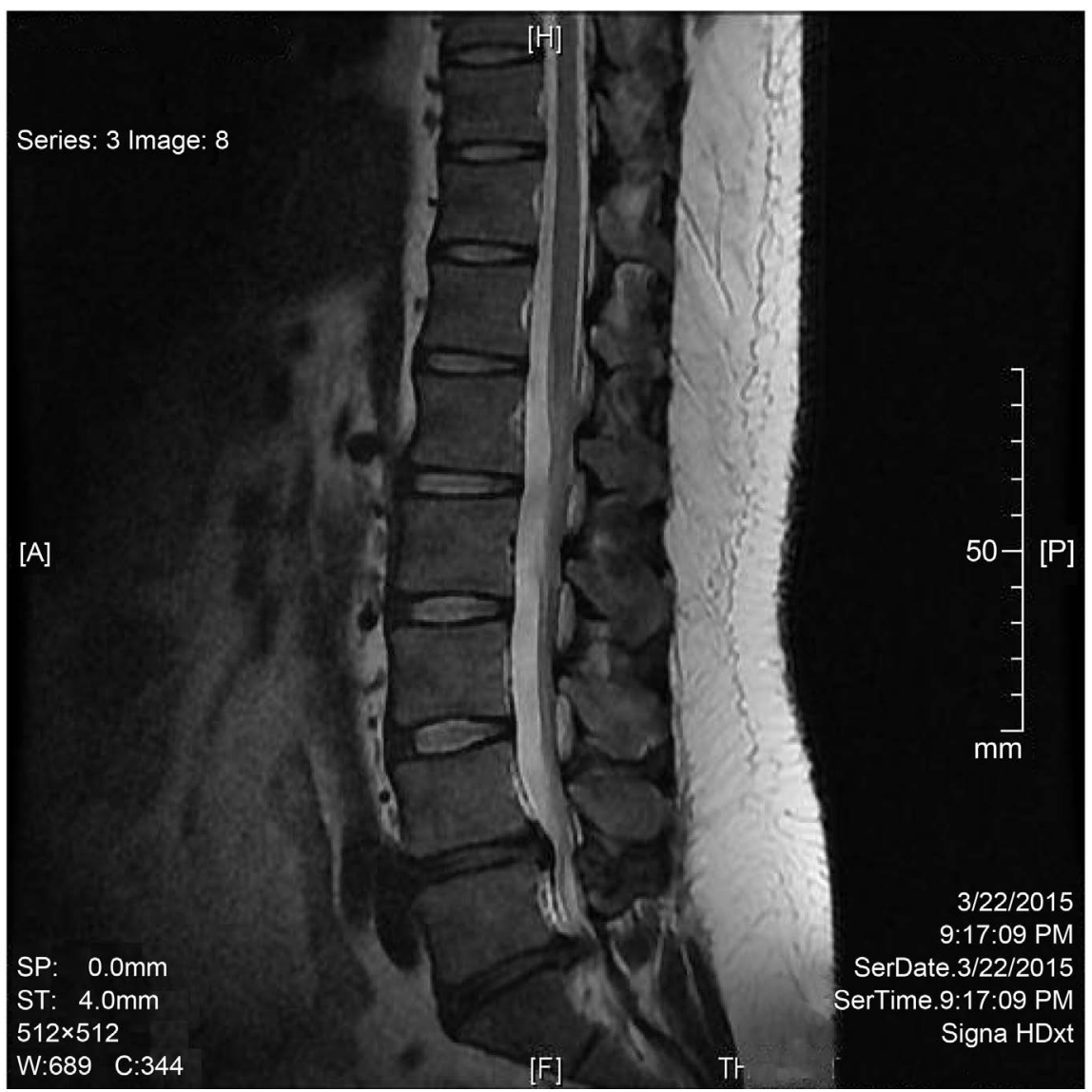

Figure 1. Magnetic resonance image of the lumbar spine.

\subsection{Histology Investigations}

Histology slides were made from the paraffin embedded excised intervertebral disc tissue Haematoxylin and Eosin staining ( $\mathrm{H}$ and $\mathrm{E}$ ) was performed on the slides.

\subsection{Management, Follow up and Prognosis}

Patient was requested to attend the follow up clinics every 3 months for a period of one year after the surgery. No inflammation or infection was observed during the follow up examination. Further there was no resurgence of the herniated intervertebral disc. Prognosis was good.

\section{Discussion}

Infections caused by Gemella morbillorum in central nervous system are scarce, but it is a commensal flora in upper respiratory tract, female genital tract, and intestinal tract. Although there are few reported studies in relation to brain abscesses; no studies have been reported on Gemella morbillorum in relation to disc herniation. Garcia-Borders, Luis et al. have reported on spontaneous pyogenic spondylodiscitis and epidural abscess in vertebral fracture [4].

Gemella morbillorum is a Gram positive, anaerobic, non-motile, non-spore forming catalase negative coccus. It was initially known as Streptococcus mor- 
billorum, but DNA homology, physiological characteristics and 16S RNA catalogue confirms it to the present genus [5].

Studies have stated that poor oral hygiene, dental procedures, colon diseases and invasive procedures could predispose to Gemella infection. Even though this patient did not have any of the above mentioned diseases, he has undergone an invasive lumbar spine surgery.

Laboratory investigations revealed that, full blood count was normal (white blood cell count: $8800 / \mathrm{mm}^{3}$, neutrophil count: $4600 / \mathrm{mm}^{3}$ ). Serum calcium level was within the normal range $(10.4 \mathrm{mg} / \mathrm{dL})$ but vitamin $\mathrm{D}$ level was slightly low (23.37 ng/mL, reference value: $26.1 \mathrm{ng} / \mathrm{mL}$ ). Serum high sensitive C-reactive protein level (hs CRP) was elevated $5.1 \mathrm{mg} / \mathrm{L}$ (reference value- $3 \mathrm{mg} / \mathrm{L}$ ) this may be attributed to the presence of a less virulent microorganism causing degenerative or inflammatory changes. In this study only degenerated collagens were present in $\mathrm{H} \& \mathrm{E}$ stain of the removed disc as presented in Figure 2, but predominant inflammatory changes were not observed. Other studies have also reported elevated hs CRP in subjects who were positive for the presence of microorganism. They have stated this might be due to either degeneration or inflammation around the disc material. Alistair et al. have also reported that inflammation seen around the nerve roots of sciatica patients might be due to microbial infection [3].

According to Rollason et al., among the anaerobic cultures harvested after discectomy of 64 patients with lumbar disc herniation, 38\% had Propionibacterium acnes in their excised herniated disc tissues. Studies have reported that bacteria can cause modic changes in adjacent vertebra and end plates promoting disc herniation when compared to others without bacterial isolation [6]. In the present study this was the second lumbar discectomy of this patient, the presence of Gemella morbillorum could have led to the changes in vertebral end plate and disc herniation. Further absences of anaerobic microorganisms in the skin

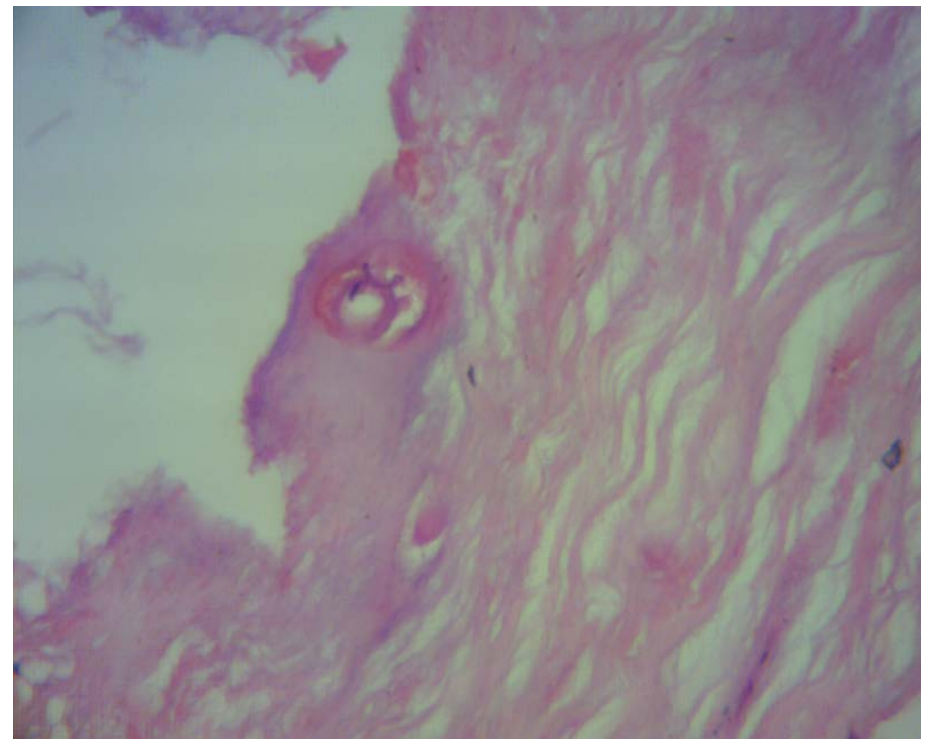

Figure 2. H \& E stain of the removed intervertebral disc portion (40x). 
scraping and muscle biopsy rule out the possibility of contamination.

Current treatment for back pain includes physiotherapy, pain killers and surgical invasion. Studies have found that treatment with antibiotics have lessened the pain symptoms related to disc herniation. Studies have found that antibiotic protocol was significantly effective than placebo in decreasing the pain and disability among disc herniated patients [7]. Therefore findings of this study further provide strong evidence that future treatment regimens with antibiotics would be a positive impact.

In this study the confirmation and identification of Gemella morbillorum was done by RapID ANA II ID kit. 16 S RNA sequencing is considered as a better method; but high cost and limited facilities were the limitations in this study.

\section{Conclusion}

This is the first case to report isolation of Gemella morbillorum in the tissue samples of a herniated lumbar disc. Findings of the present study further add to the available literature on the role of microbes in lumbar disc herniation and future treatment regimens with antibiotics.

\section{Acknowledgements}

Financial assistance by University Grants Commission, Sri Lanka (Grant No. UGC/DRIC/PG/2013), Medical Research Institute, Sri Lanka (Grant No. 522014) \& University Grant-University of Sri Jayewardenepura, Sri Lanka (Grant No. ASP/01/RE/MED/2015/47).

\section{Conflicts of Interest}

The authors report no conflicts of interest. The authors alone are responsible for the content and writing of the paper.

\section{Ethical Approval}

Ethical clearance for this study was approved by Ethics Review Committee, Faculty of Medical Sciences, University of Sri Jayewardenepura, Sri Lanka.

\section{References}

[1] Patel, N. (2002) Surgical Disorders of the Thoracic and Lumbar Spine: A Guide for Neurologists. Journal of Neurology, Neurosurgery \& Psychiatry, 73, i42-i48.

[2] Rollason, J., et al. (2013) Genotypic and Antimicrobial Characterisation of Propionibacterium acnes Isolates from Surgically Excised Lumbar Disc Herniations. BioMed Research International.

[3] Stirling, A., et al. (2001) Association between Sciatica and Propionibacterium acnes. The Lancet, 357, 2024-2025. https://doi.org/10.1016/S0140-6736(00)05109-6

[4] García-Bordes, L., et al. (2010) A Unusual Case of Spondylodiscitis. Spine, 35, E167E171. https://doi.org/10.1097/BRS.0b013e3181be5ea2

[5] Kilpper-Bälz, R. and Schleifer, K. (1988) Transfer of Streptococcus morbillorum to the Genus Gemella as Gemella morbillorum comb. nov. International Journal of 
Systematic and Evolutionary Microbiology, 38, 442-443.

https://doi.org/10.1099/00207713-38-4-442

[6] Hirose, Y., et al. (2008) A Functional Polymorphism in THBS2 That Affects Alternative Splicing and MMP Binding Is Associated with Lumbar-Disc Herniation. The American Journal of Human Genetics, 82, 1122-1129.

https://doi.org/10.1016/j.ajhg.2008.03.013

[7] Albert, H.B., et al. (2013) Antibiotic Treatment in Patients with Chronic Low Back Pain and Vertebral Bone Edema (Modic Type 1 Changes): A Double-Blind Randomized Clinical Controlled Trial of Efficacy. European Spine Journal, 22, 697-707. https://doi.org/10.1007/s00586-013-2675-y

Submit or recommend next manuscript to SCIRP and we will provide best service for you:

Accepting pre-submission inquiries through Email, Facebook, LinkedIn, Twitter, etc. A wide selection of journals (inclusive of 9 subjects, more than 200 journals) Providing 24-hour high-quality service User-friendly online submission system Fair and swift peer-review system Efficient typesetting and proofreading procedure Display of the result of downloads and visits, as well as the number of cited articles Maximum dissemination of your research work

Submit your manuscript at: http://papersubmission.scirp.org/

Or contact jbm@scirp.org 